\title{
Erratum to: Effect of the modulation of leucine zipper tumor suppressor 2 expression on proliferation of various cancer cells functions as a tumor suppressor
}

\author{
Jong Myung Kim $\cdot$ Ji Sun Song $\cdot$ Hyun Hwa Cho $\cdot$ \\ Keun Koo Shin $\cdot$ Yong Chan Bae $\cdot$ Byung Ju Lee • \\ Jin Sup Jung
}

Published online: 6 December 2011

(C) Springer Science+Business Media, LLC. 2011

\section{Erratum to: Mol Cell Biochem (2011) 346:125-136 \\ DOI 10.1007/s11010-010-0599-y}

In the Acknowledgements of the original article, the grant number was incorrectly stated. The correct acknowledgement should read as follows: This study was supported by a grant (E00027) of National Research Foundation (NRF). Everything else in the article remains correct.

The online version of the original article can be found under doi: 10.1007/s11010-010-0599-y.

J. M. Kim

MD-PhD Program, School of Medicine, Pusan National

University, Yangsan, Korea

J. M. Kim · J. S. Song · H. H. Cho - K. K. Shin · J. S. Jung ( $ه)$ Department of Physiology, School of Medicine, Pusan National

University, Beomeo-ri, Mulgeum-eup, Yangsan-si,

Gyeongsangnam-do 626-870, Korea

e-mail: jsjung@pusan.ac.kr

J. M. Kim · J. S. Song · H. H. Cho · K. K. Shin · J. S. Jung

Medical Research Center for Ischemic Tissue Regeneration,

School of Medicine, Pusan National University, Yangsan, Korea

Y. C. Bae

Department of Plastic Surgery, School of Medicine,

Pusan National University, Yangsan, Korea

B. J. Lee

Department of Otolaryngology, School of Medicine,

Pusan National University, Yangsan, Korea

J. S. Jung

Medical Research Institute, Pusan National University,

Yangsan, Korea 